\title{
The Effects of a Six-Week HIIT Program on CVD Risk Factors in Sedentary Individuals
}

Jenna N. Domblesky, Shayne T. Rockey, Nicholas M. Hoster, Melissa A. Reed, and Melissa A. Whidden. West Chester University, West Chester, PA

Regular physical activity is linked to improved cardiovascular (CV) health. High intensity interval training (HIIT) is a type of CV exercise that involves interchanging intervals of high intensity exercise (usually $80-100 \%$ of maximum heart rate) with lower intensity recovery periods. PURPOSE: The purpose of this study was to assess the effect of a six-week HIIT program on modifiable cardiovascular disease (CVD) risk factors. METHODS: Total cholesterol (TC), high-density lipoprotein (HDL) cholesterol, low-density lipoprotein (LDL) cholesterol, fasting plasma glucose (FPG), blood pressure (BP), resting heart rate (RHR), and body fat percentage (BF \%) were assessed before, halfway through, and after the six-week program in nine sedentary young adults. Subjects performed three sessions per week for six weeks at a work-to-rest (W:R) ratio of 1:4, where they were required to sprint and walk.

RESULTS: There was a significant increase in FPG from the pre-program to the post-program assessment $(P=0.03)$. There was also a significant decrease in diastolic BP (DBP) $(P=0.03)$ and RHR $(P=0.04)$ from the pre-program to post-program assessment. CONCLUSIONS: These data suggest that HIIT is effective in significantly reducing DBP and RHR. However, a sixweek, 18 session HIIT program at a 1:4 W:R ratio may not be effective for reducing the risk for CVD through idealizing blood lipids, SBP, body composition, or anthropometry measurements in this population. This study contributes to the necessity to find an optimal HIIT program length, training session duration, and $\mathrm{W}: \mathrm{R}$ ratio to help establish the most advantageous training program to reduce the risk of CVD.

Supported by the College of Health Sciences Student-Faculty Research Award 